\title{
VISION-BASED RELATIVE NAVIGATION FOR FORMATION FLYING OF SPACECRAFT
}

\author{
Roberto Alonso, $^{*}$ John L. Crassidis ${ }^{\dagger}$ and John L. Junkins ${ }^{\ddagger}$ \\ Department of Aerospace Engineering \\ Texas A\&M University \\ College Station, TX 77843-3141
}

\begin{abstract}
The objective of this paper is to develop a robust and efficient approach for relative navigation and attitude estimation of spacecraft flying in formation. The approach developed here uses information from a new optical sensor that provides a line of sight vector from the master spacecraft to the secondary satellite. The overall system provides a novel, reliable, and autonomous relative navigation and attitude determination system, employing relatively simple electronic circuits with modest digital signal processing requirements and is fully independent of any external systems. State estimation is achieved through an optimal observer design, which is analyzed using a Lyapunov and contraction mapping approach. Simulation results indicate that the combined sensor/estimator approach provides accurate relative position and attitude estimates.
\end{abstract}

\section{Introduction}

Spacecraft formation flying is an evolving technology with many possible applications, such as long baseline interferometry, stereographic imaging, synthetic apertures, and distinguishing spatial from temporal magnetospheric variations. A significant advantage of distributed spacecraft platforms over a single multifunctional spacecraft is that single point failures can be rectified through replacement of cheaper and smaller spacecraft to maintain mission capability, thus providing a more reliable and robust system. Many missions (in particular interferometry missions) rely on precise relative position and attitude knowledge in order to maintain mission requirements.

\footnotetext{
* Graduate Student of Aerospace Engineering.

$\dagger$ Assistant Professor of Aerospace Engineering. Senior Member AIAA.

${ }^{\ddagger}$ George J. Eppright Chair Professor of Aerospace Engineering. Fellow AIAA.

Copyright (C) 2000 by the American Institute of Aeronautics and Astronautics, Inc. All rights reserved.
}

Formation flying in a satellite constellation is a special structure that involves the consideration of several bodies simultaneous in time and close in position. All of the spacecraft are typically predominately attracted by a central field from a planet, such as the Earth. The motion relative among them is an issue of active research. Among the many research issues navigation is addressed in this paper. In general, the absolute position and velocity (it is called absolute because it refers to an inertial or quasi-inertial frame) of each one can be estimated by using a Global Positioning System (GPS) receiver and/or other conventional techniques, such as radar or antenna range, rate-range, angles, etc. Absolute navigation relates each one with respect to a common fixed frame. Relative navigation seeks optimal estimates for the position and velocity of one satellite relative to the other one. To date, most research studies into determining both absolute and relative positions and attitudes between vehicles in a formation have involved using GPS, ${ }^{1}$ which restricts the spacecraft formation to near-Earth applications. An application of GPS-like technology to a deep space mission has been proposed, ${ }^{2}$ but this requires extensive hardware development and is subject to the generic GPS performance-limiting effects, including multipath, geometric dilution of precision, integer ambiguity resolution, and cycle slip. In order to reduce the operational cost, size and weight of spacecraft for formation missions, new technologies are required to fill the gap implicit in standard sensors.

The vision-based navigation (VISNAV) system described in this paper comprises an optical sensor of a new kind combined with specific light sources (beacons) in order to achieve a selective or "intelligent" vision. The sensor is made up of a Position Sensing Diode (PSD) placed in the focal plane of a wide angle lens. When the rectangular silicon area of the PSD is illuminated by energy from a beacon focused by the lens, it generates electrical currents in four 
directions that can be processed with appropriate electronic equipment to estimate the energy centroid of the image. While the individual currents depend on the intensity of the light, their imbalances are weakly dependent on the intensity and are almost linearly proportional to the location of the centroid of the energy incident on the PSD. The idea behind the concept of intelligent vision is that the PSD can be used to see only specific light sources, accomplished by frequency domain modulation of the target lights and some relatively simple analog signal processing (demodulation). The light is produced by LEDs (beacons) modulated at an arbitrary known frequency while the currents generated are driven through an active filter set on the same frequency. Calculating the current imbalances then yields two analog signals directly related to the coordinates locating the centroid of that beacon's energy distribution on the PSD, in a quasi-linear fashion, and therefore to the incident direction of this light on the wide-angle lens (which gives a line of sight vector). Benefits of this configuration include: 1) Very small sensor size, 2) Very wide sensor field of view, 3) No complex/time consuming CCD signal processing or pattern recognition required, 4) Excellent rejection of ambient light interference under a wide variety of operating conditions, and 5) Relatively simple electronic circuits with modest digital signal processing (DSP) micro-computer requirements. Finally, the precision of these sensors are comparable to a star camera in establishing line of sight direction. These benefits clearly make the VISNAV system a viable sensor for relative navigation and attitude determination of spacecraft in formation. A more detailed description of the VISNAV system can be found in Ref. [3].

The dynamical equations of motion for relative position and attitude motion are highly nonlinear. The extended Kalman filter can be used to estimate the state variables, where the gains are calculated in some optimal sense to obtain the minimum variance estimate. For example, in absolute attitude estimation, implementations of the filter shown in Ref. [4] has been successfully used on several real spacecraft for many years now. The main disadvantage in the extended Kalman filter is the potential for divergence of the filter in cases where the distance between the local linearization and the true models is large, which is typically mitigated by redundant sensors on the spacecraft. A different approach is followed in this paper for relative navigation and attitude estimation using the VISNAV system, which is largely based on the work of Salcudean. ${ }^{5}$ This uses an optimal observer that ensures some kind of globally asymptotic stability. Therefore, the need for redundant systems in formations of spacecraft can be reduced.

The organization of this paper proceeds as follows. First, the basic equations for the VISNAV system are given. Then, the relative attitude equations are de- rived, followed by a derivation of the orbital equations of motion. Next, the dynamical equations are simplified by using some control design assumptions. Then, the observer is derived for relative attitude and position estimation. The stability of the observer is assessed through a Lyapunov and contraction mapping analysis. Finally, simulation results are presented.

\section{Basic Equations}

In this section the mathematical models are presented in the context of the particular problem related to relative position and attitude estimation from line of sight observations. The notation ${ }^{6}$ used in the derivations is briefly revisited for the sake of clarification. The angular velocity of the $\alpha$ frame with respect to the $\beta$ frame is represented by the physical vector $\boldsymbol{\omega}_{\beta \alpha}$ (physical denotes that the vector is independent of the frame, whereas mathematical denotes the physical vector components expressed in some frame). The vector $\boldsymbol{\omega}_{\beta \alpha}^{\gamma}$ is the mathematical vector made up of the components of $\boldsymbol{\omega}_{\beta \alpha}$ taken in the $\gamma$ frame. The derivative with respect to time is indicated by the operator $p$, where $p_{\alpha} \mathbf{R}$ is the rate of change of the vector $\mathbf{R}$ relative to the frame $\alpha$, and $p \mathbf{R}^{\alpha}$ is the time derivative of the vector expressed in the $\alpha$ frame.

\section{Measurement Equation}

Figure 1 shows the focal plane measurement of the VISNAV system for a master and secondary satellite system using one light source from a beacon (see Ref. [3] for more details). Three frames are used to describe the orientation and position of the master and secondary satellites. The first one, denoted by $\left(X_{s}, Y_{s}, Z_{s}\right)$, is fixed on the secondary satellite, with the LED beacons firmly attached to the body of the satellite, and having known positions in the $\left(X_{s}, Y_{s}, Z_{s}\right)$ frame. This frame is also the reference frame for the attitude problem. We assume that this frame is centered at the mass center of this spacecraft, and is denoted using the superscript $s$ on the mathematical vectors. The second reference system, denoted by $\left(X_{f}, Y_{f}, Z_{f}\right)$, is fixed on the master satellite, where the focal plane of the VISNAV system is located. We assume that the $Z_{f}$ axis is along the boresight, passing through the input pin hole which is at a distance $Z_{f}=+f$ from the focal plane. The axes $X_{f}$ and $Y_{f}$ are arbitrary, but fixed in the VISNAV sensor. This frame is denoted as the $f$ frame. The third frame, denoted by $\left(X_{m}, Y_{m}, Z_{m}\right)$, is fixed to the mass center of the master satellite. The position and orientation of this frame with respect to the focal frame is assumed to be known. The vectors for the master frame are identified with the superscript $m$.

The point $S$ is the origin of the frame $s$. The point $O$ is the location of each light beacon in the secondary satellite; normally there are several beacons to assure continuous tracking of the satellite and for redundancy. 


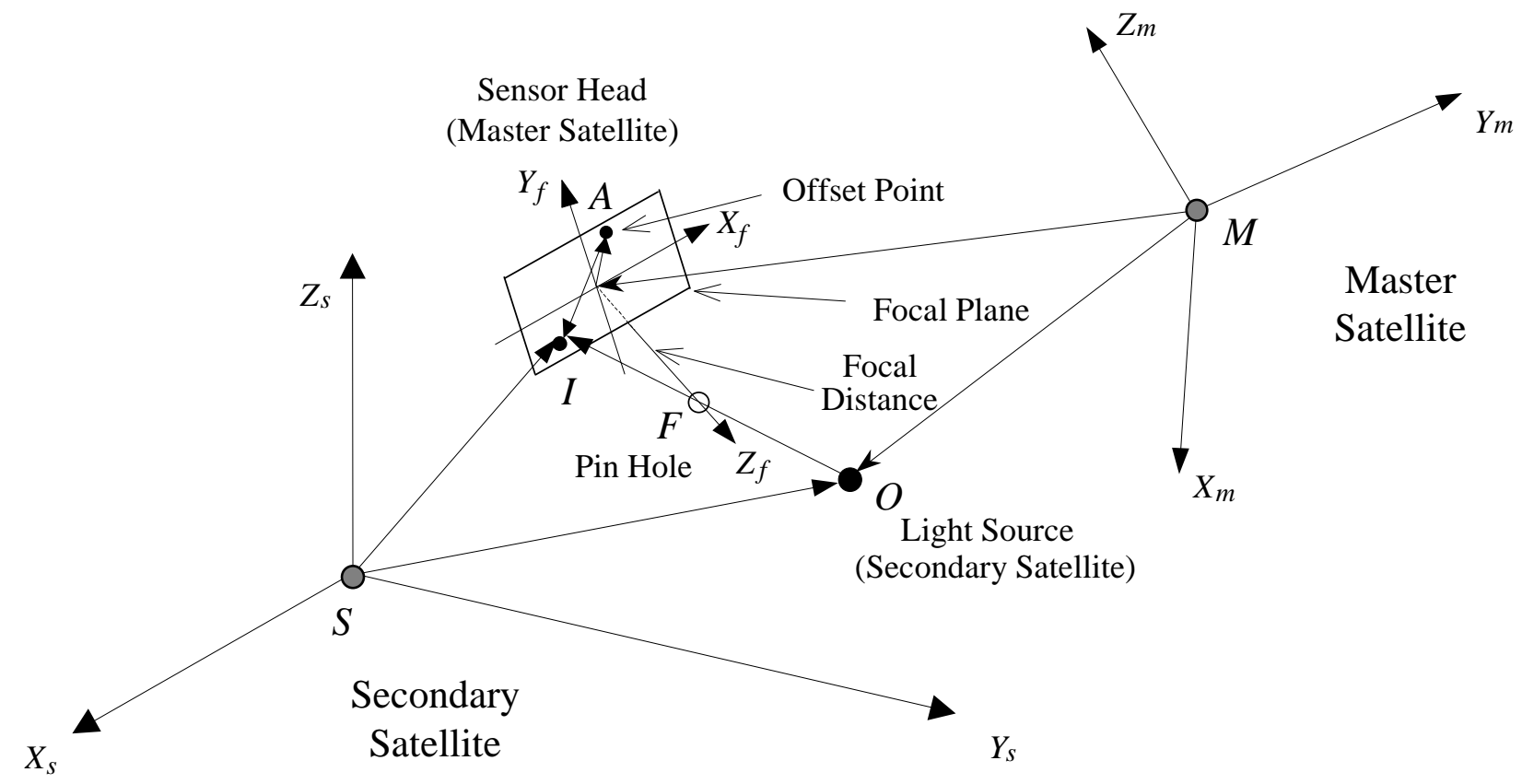

Fig. 1 Focal Plane Measurement from One Light Source

The point $I$ is sometimes referred as the image center since it is the intersection of each light beam from the beacon with the focal plane, where position of $I$ with respect to the focal reference system is used to form a line of sight observation. The point denoted as $F$ in Figure 1 is the pinhole which is coincident with the sensor principal point. Three vectors are now defined: $\overrightarrow{S O}$ (the vector from the center $S$ of the $s$ frame to the beacon location $O$ ), $\overrightarrow{S I}$ (the vector from the the center $S$ of the $s$ frame to the image center $I$ ), and $\overrightarrow{O I}$ (the vector from the beacon location $O$ to the image center $I$, with the constraint equation given by $\overrightarrow{O I}=$ $\overrightarrow{S I}-\overrightarrow{S O}$.

The orientation between the secondary and master frames is denoted by the (unknown) rotation matrix $C_{s}^{m}$ which transforms a vector expressed in the secondary frame $s$ to the primary frame $m$. The rotation matrix $C_{f}^{m}$ between the focal and the master frames is known by ground calibration. Expressing the vectors $\overrightarrow{S I}, \overrightarrow{O I}$ and $\overrightarrow{S O}$ in frame components gives the following relation ${ }^{7}$

$$
C_{s}^{m}(\overrightarrow{S I}-\overrightarrow{S O})^{s} \equiv C_{s}^{m} \mathbf{v}^{s}=\mathbf{v}^{m} \equiv(\overrightarrow{O I})^{m}
$$

where

$$
\begin{gathered}
\mathbf{v}^{s}=\frac{1}{\sqrt{\xi}}\left[\begin{array}{c}
X_{I}-x_{O} \\
Y_{I}-y_{O} \\
Z_{I}-z_{O}
\end{array}\right] \\
\xi \equiv\left(X_{I}-x_{O}\right)^{2}+\left(Y_{I}-y_{O}\right)^{2}+\left(Z_{I}-z_{O}\right)^{2}
\end{gathered}
$$

and $\left(X_{O}, Y_{O}, Z_{O}\right)$ represents the known beacon location, and $\left(X_{I}, Y_{I}, Z_{I}\right)$ is the unknown position with respect to the secondary satellite. The measurements $x_{I}$ and $y_{I}$ in the focal frame can be expressed in unit vector form by

$$
\mathbf{v}^{f}=\frac{1}{\sqrt{x_{I}^{2}+y_{I}^{2}+f^{2}}}\left[\begin{array}{c}
x_{I} \\
y_{I} \\
-f
\end{array}\right]
$$

where $f$ is the known focal distance. This unit vector in the master frame is expressed using the fixed rotation matrix between the sensor plane frame and the master satellite reference frame, with $\mathbf{v}^{m}=C_{f}^{m} \mathbf{v}^{f}$. A bias offset in the measurement is also accounted for in the model (denoted by $A$ in Figure 1). The bias vector is a constant error vector induced by an unbalance of the horizontal and vertical gains in the focal plane detector relative to the particular coordinate system associated with the detector at calibration. This vector is denoted by $\mathbf{v}_{a}$ and is normally referenced in the focal plane frame:

$$
\mathbf{v}_{a}^{m}=C_{f}^{m} \mathbf{v}_{a}^{f}=C_{f}^{m}\left[\begin{array}{c}
x_{a} \\
y_{a} \\
0
\end{array}\right]^{f}
$$

Finally, the measurement equation for each light source from a beacon, placed on the secondary satellite, is as follows:

$$
\mathbf{v}_{j}^{m}=C_{s}^{m} \mathbf{v}_{j}^{s}+\mathbf{v}_{a}^{m} \text { for } j=1, \ldots, N
$$

where $N$ is the number of LED beacons.

Small separations between light beams from multiple LEDs reduces the discrimination of each beacon, which ultimately produces a dilution of precision in 
the position and attitude solution. A larger distance between the satellites also produces a dilution of precision since the beacons ultimately approach angular co-location. If the relative position between satellites is known then only two non-colinear line of sight vectors are required to determine an attitude solution. In a similar fashion for the position navigation only problem, where the satellite is considered to be a "mass point" (in other words without attitude), two line of sight vectors are only required. A covariance analysis shows that when the relative position and attitude both are unknown then two line of sight vectors provide only one axis of attitude and one axis of position

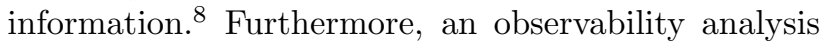
using two line of sight observations indicates that the beacon that is closest to the target provides the most attitude information but has the least position information, and the beacon that is farthest to the target provides the most position information but has the least attitude information. In order to find a deterministic solution for the position and velocity at least four vector observations are required.

\section{Relative Attitude Equations}

In this section the governing equations for the relative attitude dynamics between two bodies are reviewed. The dynamical equations presented here are derived using non-inertial reference frames, however only minor changes are required from the standard formulation. ${ }^{6}$ Starting from Eq. (5) and taking derivative of each vector with respect to the same frame in which they are expressed gives the following expressions

$$
\begin{aligned}
p \mathbf{v}^{m} & =C_{s}^{m} p \mathbf{v}^{s}+p C_{s}^{m} \mathbf{v}^{s} \\
& =C_{s}^{p}\left(p \mathbf{v}^{s}+C_{m}^{s} p C_{s}^{m} \mathbf{v}^{s}\right)
\end{aligned}
$$

The bias in Eq. (5) is considered to be a constant, so it's derivative is zero. The same expression in Eq. (6) can be derived by the application of the transport theorem, which yields the following expressions

$$
\begin{aligned}
& p_{m} \mathbf{v}=p_{s} \mathbf{v}+\boldsymbol{\omega}_{m s} \times \mathbf{v} \\
& p \mathbf{v}^{m}=C_{s}^{m}\left(p \mathbf{v}^{s}+\left[\boldsymbol{\omega}_{m s}^{s} \times\right] \mathbf{v}^{s}\right)
\end{aligned}
$$

where the matrix $[\cdot \times]$ denotes the cross product matrix, given by

$$
[\boldsymbol{\omega} \times] \equiv\left[\begin{array}{ccc}
0 & -\omega_{3} & \omega_{2} \\
\omega_{3} & 0 & -\omega_{1} \\
-\omega_{2} & \omega_{1} & 0
\end{array}\right]
$$

Both expressions, (6) and (7), must be equivalent. Setting these equations equal to each other yields the time rate of change of the attitude matrix, given by

$$
\begin{aligned}
C_{m}^{s} p C_{s}^{m} & =\left[\boldsymbol{\omega}_{m s}^{s} \times\right] \\
p C_{s}^{m} & =C_{s}^{m}\left[\boldsymbol{\omega}_{m s}^{s} \times\right]=-\left[\boldsymbol{\omega}_{s p}^{m} \times\right] C_{s}^{m}
\end{aligned}
$$

The relative attitude dynamics are described by the expression in Eq. (9) in terms of attitude matrix and the angular velocity between both frames.

We now write the expression in Eq. (9) in terms of the corresponding quaternions. ${ }^{9}$ Toward this end, the quaternion is expressed as, $\mathbf{q}_{m}^{s}=\left[\begin{array}{ll}\mathbf{e}^{T} \sin \frac{\alpha}{2} & \cos \frac{\alpha}{2}\end{array}\right]^{T}$, where $\mathbf{e}$ is the eigenaxis between both frames and $\alpha$ is the rotation angle measured from frame $m$ to frame $s$. The quaternion is a vector and has the same components in both the $m$ and $s$ frames, and can be expressed in any external frame as an arbitrary (i.e. general) vector. This has an advantage over the rotation matrix formulation, which is fixed to the reference system $s$ and $m$ in this case. This property of the quaternion is used later.

An infinitesimal rotation is expressed in terms of the quaternion as $d \mathbf{q}_{m}^{s}=1+\frac{1}{2} \boldsymbol{\omega}_{s m} d t$, where $d t$ is the time differential. Multiplying by the quaternion $\mathbf{q}_{m}^{s}$ and taking the first-order infinitesimal part, the following differential equation is given

$$
\begin{aligned}
p \mathbf{q}_{m}^{s} & =\frac{1}{2} \mathbf{q}_{m}^{s} \otimes \boldsymbol{\omega}_{s m}^{m} \\
& =\frac{1}{2}\left[\begin{array}{ccc}
\varrho & \vdots & q_{o} I_{3 \times 3}+[\boldsymbol{\varrho} \times] \\
\ldots & \vdots & \ldots \ldots \ldots \ldots \ldots \\
q_{o} & \vdots & -\boldsymbol{\varrho}^{T}
\end{array}\right]\left[\begin{array}{c}
\boldsymbol{\omega}_{s m} \\
\ldots \\
0
\end{array}\right] \\
& =\frac{1}{2}\left[\begin{array}{c}
q_{o} \boldsymbol{\omega}_{s m}+[\boldsymbol{\varrho} \times] \boldsymbol{\omega}_{s m} \\
\ldots \ldots \ldots \ldots \\
-\varrho^{T} \cdot \boldsymbol{\omega}_{s m}
\end{array}\right]
\end{aligned}
$$

where the quaternion $\mathbf{q}_{m}^{s}$ is decomposed into a scalar and a vector part as $\mathbf{q}_{m}^{s}=\left[\begin{array}{ll}\left(\varrho_{m}^{s}\right)^{T} & q_{0}\end{array}\right]^{T}$, and $[\boldsymbol{\varrho} \times] \in$ $\Re^{3 \times 3}$ is the skew symmetric matrix which represents the cross product $\varrho \times(\cdot)=[\boldsymbol{\varrho} \times](\cdot)$. Both the attitude matrix and quaternion formulations will be used in the definition of the observer feedback error, but the quaternion formulation is used in the actual implementation of the observer.

\section{Relative Navigation Equations}

From basic orbit theory, ${ }^{10}$ the equations of motion are written assuming that each satellite is referenced with respect to the same inertial frame. The vectors are described in the Figures 2 and 3:

$$
\begin{aligned}
p_{i}^{2} \mathbf{R}_{s} & =-G m_{e} \frac{\mathbf{R}_{m}+\mathbf{r}}{\left\|\mathbf{R}_{m}+\mathbf{r}\right\|^{3}}+\mathbf{a}_{s} \\
p_{i}^{2} \mathbf{R}_{m} & =-G m_{e} \frac{\mathbf{R}_{m}}{\left\|\mathbf{R}_{m}\right\|^{3}}+\mathbf{a}_{m}
\end{aligned}
$$

where $G$ is the universal constant of gravitation and $m_{e}$ is the mass of the Earth. The relative orbit is described by the difference between both vectors, $\mathbf{r}=\mathbf{R}_{m}-\mathbf{R}_{s}$. Taking derivatives with respect to the 


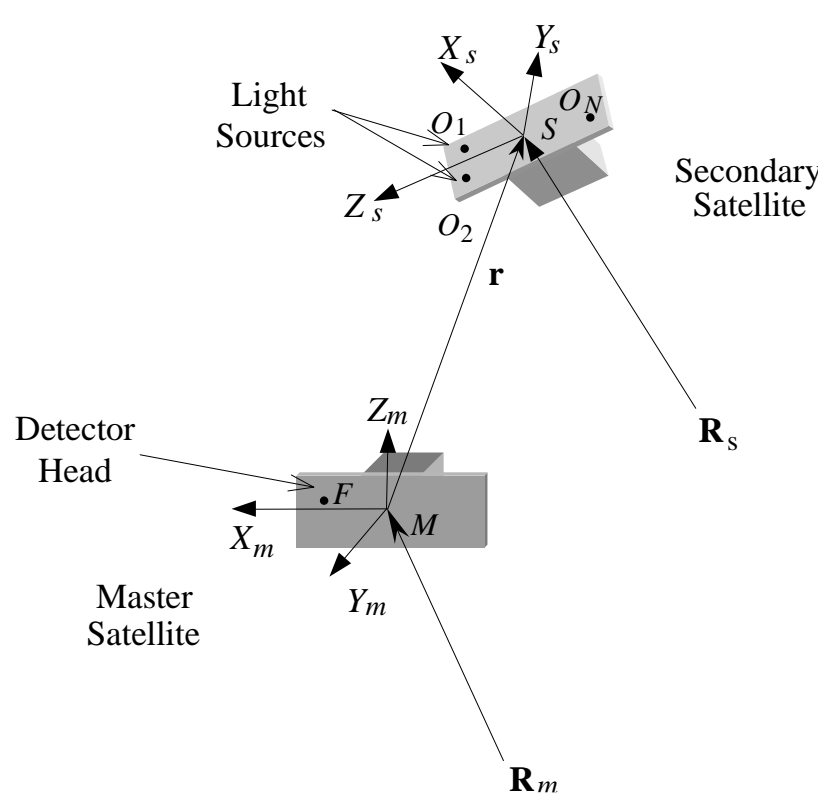

Fig. 2 Formation Flying Geometry.

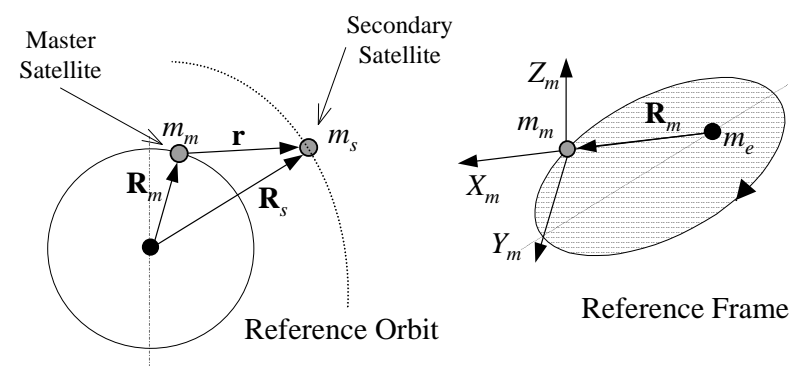

Fig. 3 Relative Navigation: the master and the secondary satellite orbits.

inertial frame, the following equation is obtained

$$
p_{i}^{2} \mathbf{r}=-\mu_{e}\left[\frac{\mathbf{R}_{m}+\mathbf{r}}{\left\|\mathbf{R}_{m}+\mathbf{r}\right\|^{3}}-\frac{\mathbf{R}_{m}}{\left\|\mathbf{R}_{m}\right\|^{3}}\right]+\Delta \mathbf{a}
$$

where $\mu_{e} \equiv G m_{e}$. The quantity $\Delta \mathbf{a}=\left(\mathbf{a}_{s}-\mathbf{a}_{p}\right)$ is the differential disturbance between both satellites. It collects the higher order gravitational potential terms (J2, $\mathrm{J} 3$, etc), and the nonconservative forces (such as drag, solar pressure, etc.) applied to the satellites. Only the differential terms are considered, generated by a difference in altitude, in mass, in exposed area, etc., which in general reduces the magnitude of the absolute perturbation. For example, similar satellites with large solar arrays orbiting near each other cannot have relative drag forces or torques between them, even for the case where each individual satellite is subject to a strong drag perturbation. In other words both satellite have orbits that are decaying at the same rate.

The differential equation for the relative navigation problem, which is described by Eq. (12), can be simplified if an appropriate reference system, other than the inertial frame, is used to express the relative position and velocity vectors. If the master satellite position vector is written as $\mathbf{R}_{m}=R_{m}[1,0,0]^{T}$, the expression can be simplified. The frame with this property is the Local Vertical Local Horizontal (LVLH) reference frame, ${ }^{11}$ which is widely used to reference Earth Pointing satellites. The LVLH frame is centered at the mass center of the master satellite, and the axes have the following orientation: the $X$ axis is along the position vector, the $Z$ axis is normal to the motion plane (strictly speaking, the osculating plane), and the $Y$ axis is defined to complete a right hand orthogonal reference frame.

In special cases, to describe the relative motion a non-orthogonal reference frame is chosen in order to place the velocity vector continuously along the $Y$ axis, and the position along the $X$ axis (this condition is valid only for circular orbits using the LVLH frame). This simple fact gives a very efficient representation of the state vector at any time, but it requires an extra non-orthogonal transformation at every step time, which can be expensive from a computational point of view. This type of representation is convenient for the case of highly eccentric orbits, which may not be common in low altitude satellite constellations. In addition the time derivative can be replaced by the derivative with respect to the true anomaly, which is a valid transformation because it is an increasing time function. ${ }^{12}$

By using the transport theorem, the left side of Eq. (12) is differentiated with respect to the LVLH frame, which is noted from now on as $m$ for simplicity. Both frames may of may not be coincident, but they must have a fixed transformation between them. Because the $m$ frame has axes with free orientation, the LVLH represents just one of the possible frame choices. In this paper both the $m$ and LVLH frames are the same so that

$$
\begin{aligned}
p_{i}^{2} \mathbf{r} & =p_{m}^{2} \mathbf{r}+2 \boldsymbol{\omega}_{i m} \times p_{b} \mathbf{r}+p_{m} \boldsymbol{\omega}_{i m} \times \mathbf{r} \\
& +\boldsymbol{\omega}_{i m} \times\left(\boldsymbol{\omega}_{i m} \times \mathbf{r}\right)
\end{aligned}
$$

where $\boldsymbol{\omega}_{i m}$ is the angular velocity of the master frame with respect to the inertial frame and takes values only along the $Z$ axis of the $m$ frame, i.e., $\boldsymbol{\omega}_{i m}^{m}=[0,0, \omega]^{T}$.

The angular acceleration, $p \boldsymbol{\omega}_{i m}^{m}$, is denoted for simplicity as $\dot{\boldsymbol{\omega}}_{i m}^{m}$. This is expressed in the $m$ frame as $\dot{\boldsymbol{\omega}}_{i m}^{m}=[0,0, \dot{\omega}]^{T}$. The vector in Eq. (13) is decomposed in $m$ frame components and takes the final expression given by

$$
\begin{aligned}
{\left[\begin{array}{c}
\ddot{x} \\
\ddot{y} \\
\ddot{z}
\end{array}\right] } & =-\mu\left[\begin{array}{c}
\frac{R_{m}+x}{\left\|\mathbf{R}_{m}+\mathbf{r}\right\|_{y}^{3}}-\frac{R_{m}}{\left\|\mathbf{R}_{m}\right\|^{3}} \\
\frac{\left\|\mathbf{R}_{m}+\mathbf{r}\right\|^{3}}{\left\|\mathbf{R}_{m}+\mathbf{r}\right\|^{3}}
\end{array}\right]-\dot{\omega}\left[\begin{array}{c}
-y \\
x \\
0
\end{array}\right] \\
& -\omega\left[\begin{array}{c}
-2 \dot{y}-\omega x \\
2 \dot{x}-\omega y \\
0
\end{array}\right]+\left[\begin{array}{l}
\Delta a_{x} \\
\Delta a_{y} \\
\Delta a_{z}
\end{array}\right]
\end{aligned}
$$


The forcing part along the $X$ axis component has the following structure: $\left[f\left(\mathbf{R}_{m}+\mathbf{r}_{12}\right)-f\left(\mathbf{R}_{m}\right)\right]^{m}$ which is not robust from a numerical point of view. This expression is maintained for compactness and will be used in the observer analysis, but for practical implementations it is convenient to re-write it avoiding the subtraction of two large numbers. ${ }^{10}$ Equation (14) expresses the dynamical model for relative navigation between the secondary satellite with respect to the master satellite.

We note that the number of master satellite orbit parameters computed on the ground and to be used in Eq. (14) is at most 3. For the general case, the magnitude $R_{m}$, the angular velocity $\omega$, and angular acceleration $\dot{\omega}$ are just needed. For the special case involving circular orbits, only the position magnitude is necessary.

\section{Equation for the Relative Dynamics}

In the attitude problem, Euler's equation or the measured gyro outputs are the starting point for the derivation of the rotational dynamics equation to obtain the angular velocity between the inertial frame and a body frame. For the relative attitude problem, Euler's equation must be applied in a differential mode, similar in fashion as the orbit case. However, we seek an expression without an additional "third" frame (inertial one included), in addition to the $m$ and the $s$ frames, so that the system is independent of the extra reference frame's choice. In other words, the relative navigation and the relative attitude must be a function only of the definition of the master and secondary frames and completely independent of the particular choice of the inertial frame or any other frame other than $m$ and $s$. This simple fact is common in control theory, where the error or its derivative is only defined by the current and the desired state independent of any other frame choice.

In the two body problem previously derived, the equation for $\mathbf{r}$ is very accurate because it is supported by well known models for almost all involved forces in hand, with any remaining small perturbation bounded. In the relative attitude dynamics the presence of internal torques, which are normally unmodeled with an unbounded time integral, plays an important role in the model equations. We assume that each satellite in the constellation has an attitude control subsystem able to maintain the desired satellite orientation inside of some allowable bound. The last hypothesis is a qualitative one. We assume that the measurements are available frequently enough to use simpler propagation models (to be derived) as a function of the sampling interval.

The derivation begins with the computation of the angular velocity between the secondary and the inertial frame, denoted by the subscript $i$ :

$$
\boldsymbol{\omega}_{i s}=\boldsymbol{\omega}_{i m}+\boldsymbol{\omega}_{m s}
$$

Taking derivatives with respect to the inertial frame of both sides of Eq. (15), and then applying Euler's equation on both sides, the following is obtained

$$
\begin{gathered}
p_{i} \boldsymbol{\omega}_{i s}=p_{i} \boldsymbol{\omega}_{i m}+p_{i} \boldsymbol{\omega}_{m s} \\
p_{s} \boldsymbol{\omega}_{i s}=p_{m} \boldsymbol{\omega}_{i m}+p_{m} \boldsymbol{\omega}_{m s}+\boldsymbol{\omega}_{i m} \times \boldsymbol{\omega}_{m s} \\
\frac{\mathbf{T}_{c s}+\mathbf{T}_{d s}-\boldsymbol{\omega}_{i s} \times I_{s} \boldsymbol{\omega}_{i s}}{I_{s}}=\boldsymbol{\omega}_{i m} \times \boldsymbol{\omega}_{m s} \\
+p_{m} \boldsymbol{\omega}_{m s}+\frac{\mathbf{T}_{c m}+\mathbf{T}_{d m}-\boldsymbol{\omega}_{i m} \times I_{m} \boldsymbol{\omega}_{i m}}{I_{m}}
\end{gathered}
$$

where $\mathbf{T}_{c m}$ and $\mathbf{T}_{c s}$ are the control torques applied to the master and the secondary satellite, respectively, $\mathbf{T}_{d m}$ and $\mathbf{T}_{d s}$ are the disturbance torques on each satellite, and $\mathbf{I}_{m}$ and $\mathbf{I}_{s}$ are the respective inertia tensors.

The controller of each satellite is designed to maintain the attitude error and the angular velocity error between the body frame and the desired frame, which is denoted by $d$, to within a small tolerance. Denoting the angular velocity between the secondary frame and the desired frame as $\boldsymbol{\omega}_{d s}$, the following equations are directly derived

$$
\begin{aligned}
\boldsymbol{\omega}_{i s} & =\boldsymbol{\omega}_{i d}+\boldsymbol{\omega}_{d s} \\
p_{s} \boldsymbol{\omega}_{i s} & =p_{s} \boldsymbol{\omega}_{i d}+p_{s} \boldsymbol{\omega}_{i s}
\end{aligned}
$$

In general $p_{s} \boldsymbol{\omega}_{i d} \approx \mathbf{0}$, because the angular velocity between the desired and inertial frame is constant or smooth enough to consider it to be negligible. Therefore, the first order approximation of Eq. (18) is given by $p_{s} \boldsymbol{\omega}_{i s}=p_{s} \boldsymbol{\omega}_{d s}+o\left(\left\|p_{s} \boldsymbol{\omega}_{i d}\right\|\right)$. To maintain the satellite on the desired manifold, where $p_{s} \boldsymbol{\omega}_{d s}=\mathbf{0}$, the desired torque should be equal to the sum of the equivalent torque and an extra term to make $p_{s} \boldsymbol{\omega}_{d s}$ converge to zero in finite time.

For the secondary satellite, the dynamics to drive the angular rate error to the desired manifold is driven by the following differential equation

$$
I_{s} p_{s} \boldsymbol{\omega}_{d s}=-T_{c s}-T_{d s}+\boldsymbol{\omega}_{i s} \times I_{s} \boldsymbol{\omega}_{i s}-\lambda_{s} \boldsymbol{\omega}_{d s}
$$

where the constant $\lambda_{s}$ in effect dictates a time constant, which is selected to fulfill the requirement of the control system. Similarly, for the master satellite we have

$$
\begin{aligned}
I_{m} p_{m} \boldsymbol{\omega}_{d m} & =-T_{c m}-T_{d m}+\boldsymbol{\omega}_{i m} \times I_{m} \boldsymbol{\omega}_{i m} \\
& -\lambda_{m} \boldsymbol{\omega}_{d m}
\end{aligned}
$$

Substituting Eqs. (19) and (20) into Eq. (17) yields

$$
\begin{aligned}
p \boldsymbol{\omega}_{m s}^{m} & =-\left[\begin{array}{ccc}
I_{x}^{-1} \lambda_{x} & \omega_{d m_{z}} & -\omega_{d m_{y}} \\
-\omega_{d m_{z}} & I_{y}^{-1} \lambda_{y} & \omega_{d m_{x}} \\
\omega_{d m_{y}} & -\omega_{d m_{x}} & I_{z}^{-1} \lambda_{z}
\end{array}\right] \boldsymbol{\omega}_{m s}^{m} \\
& +\Delta \mathbf{T}^{m}+\boldsymbol{\eta}_{\omega}
\end{aligned}
$$

where $\lambda_{m}$ and $\lambda_{s}$ are chosen such that $I_{m}^{-1} \lambda_{m} \approx$ $I_{s}^{-1} \lambda_{s} \approx I^{-1} \lambda$ for each axis. This approximation is 

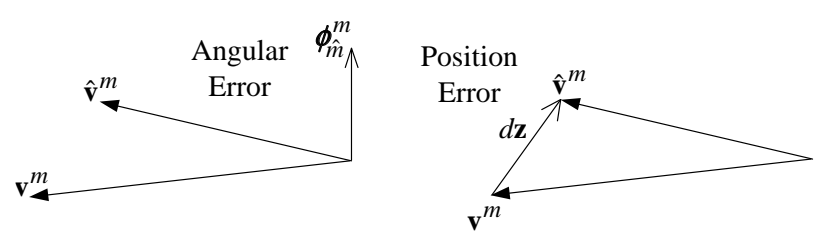

Fig. 4 Angular and Position Error Visualization

valid since the control system for each satellite will give the same response (i.e., each satellite is assumed to be controlled identically). The vector components of $\boldsymbol{\omega}_{d m}$ are known in the master frame, but they are second order terms and can typically be ignored for short time propagation. If the above approximation is the identity relation, i.e., $I_{m}^{-1} \lambda_{m}=I_{s}^{-1} \lambda_{s}=I^{-1} \lambda$, then $\Delta \mathbf{T}^{m}=\mathbf{0}$. A simple first order equation is used to model the effect of a mismatch between $I_{m}^{-1} \lambda_{m}$ and $I_{s}^{-1} \lambda_{s}$, with

$$
p \Delta \mathbf{T}^{m}=-H \Delta \mathbf{T}+\boldsymbol{\eta}_{\Delta T}
$$

where $H$ is a positive definite matrix, which is typically diagonal. The term $\boldsymbol{\eta}_{\Delta T}$ represents a Gaussian noise vector. Finally, the relative dynamics obey the following equations

$$
\begin{aligned}
\dot{\boldsymbol{\omega}}_{m s}^{m} & =\Lambda \boldsymbol{\omega}_{m s}^{m}+\Delta \mathbf{T}^{m}+\boldsymbol{\eta}_{\omega} \\
\Delta \dot{\mathbf{T}}^{m} & =-H \Delta \mathbf{T}+\boldsymbol{\eta}_{\Delta T}
\end{aligned}
$$

These are the main angular rate equations used in the observer design.

\section{Observer Design}

In this section an observer is designed to estimate the relative attitude and angular velocity as well as the relative position and the linear velocity. Because the measurements are based on the line of sight projection onto the focal plane, the angular orientation and linear position cannot be decoupled (the same is true for the angular and linear velocities). In Ref. [8] the information matrix of the attitude and position estimation errors is explicitly calculated for two line of sight observations. The information matrix is divided into four partitions, where the two main diagonal elements correspond to the attitude and position information matrices that have the identical structure if each problem (i.e., attitude or position) was considered independent of each other. The off-diagonal partitions couple the attitude and the position errors. A diagonalization (i.e., a decoupling of the attitude and position) of the information matrix occurs only in very special cases. Therefore, the entire problem which includes both attitude and position estimation is considered in the observer design.

\section{Attitude and Angular Velocity Observer}

The observer design treats the attitude portion by representing the residual (measurement minus estimate) error through a quaternion vector formulation, and treats the position portion of the residual in a straightforward position vector formulation. The angular error between the measured $\left(\mathbf{v}^{m}\right)$ and the estimated $\left(\hat{\mathbf{v}}^{m}\right)$ vectors in master frame can be "visualized" by a rotation axes normal to plane that contains both vectors. This axis $\left(\phi_{\dot{m}}^{m}\right)$ can be interpreted as the vector part of the quaternion error, and the rotation angle between both vectors is the scalar part of the quaternion. The position error $(d \mathbf{z})$ is simple vector difference between the estimated and measured vectors. Figure 4 shows both approaches.

Before continuing with this concept, the following matrix relation is first written

$$
C_{s}^{m}=C_{\hat{m}}^{m} \hat{C}_{s}^{\hat{m}}=\Delta C \hat{C}_{s}^{\hat{m}}
$$

where the estimated vector, matrix or frames are noted with the superscript $(\hat{\cdot})$, and $C_{\hat{m}}^{m} \equiv \Delta C$. The rotation error matrix between the estimated and measured quantities can be written in terms of the quaternion as $\Delta C=I+2 q_{o} \varrho^{k}+2[\boldsymbol{\varrho} \times]^{2}$. To simplify the notation this matrix is simply defined as $\Delta C \equiv(I+[\boldsymbol{\delta} \times])_{\hat{m}}^{m}$. Equation (5) can now be re-written as

$$
\mathbf{v}^{m}=(I+[\boldsymbol{\delta} \times])_{\hat{m}}^{m} \hat{C}_{s}^{\hat{m}} \hat{\mathbf{v}}^{s}
$$

where $\hat{\mathbf{v}}^{s}$ is an estimated vector, which depends on the relative position. This clearly shows the coupling of the relative navigation and attitude problems (in the standard attitude problem this vector is the assumed known reference vector, e.g., the vector to the sun or a star in an inertial frame). Equation (26) can be rewritten in residual form as

$$
\mathbf{v}^{m}-\hat{\mathbf{v}}^{m}=[\boldsymbol{\delta} \times]_{\hat{m}}^{m} \hat{\mathbf{v}}^{\hat{m}}
$$

Using the multiplicative property of the cross product matrix the right hand side of Eq. (27) can be expressed in a more convenient form as

$$
\hat{\mathbf{v}}^{m}-\mathbf{v}^{m}=[\hat{\mathbf{v}} \times]^{\hat{m}}\left(\boldsymbol{\phi}_{\hat{m}}^{m}\right)^{\hat{m}}
$$

where the vector $\phi_{\hat{m}}^{m}$ is expressed as the vector part of a quaternion in any frame. As stated previously this is an advantage of using the quaternion parameterization over the rotation matrix in the observer. The left hand side of Eq. (28) is denoted by $d \mathbf{z} \equiv \hat{\mathbf{v}}^{m}-\mathbf{v}^{m}$ for simplicity.

The number of measured line of sight vectors is generally greater than one, and the processing of this information can be done in the least square sense. Each estimated vector cross product is stacked into a matrix as

$$
\hat{V}_{\hat{m}}=\left[\begin{array}{c}
{\left[\hat{\mathbf{v}}_{1} \times\right]^{\hat{m}}} \\
\vdots \\
{\left[\hat{\mathbf{v}}_{N} \times\right]^{\hat{m}}}
\end{array}\right]
$$

In this case the pseudoinverse is computed using all available information. Therefore, the quaternion error 
is computed by

$$
\hat{V}_{\hat{m}}^{+} d \mathbf{z}=\phi_{s}^{m} \rightarrow\left[\delta \mathbf{q}, \delta q_{o}\right]
$$

where $\hat{V}_{\hat{m}}^{+}$is the pseudoinverse of $\hat{V}_{\hat{m}}$. The computation of the quaternion error is comparable to the algorithm presented in Ref. [4]. In the approach of [4] the scalar part of the quaternion $\left(\delta q_{o}\right)$ is assumed to always be equal to +1 . However, the scheme presented in this section maintains all four elements of the quaternion error because the sign of the scalar part is used in the design of the observer.

The nonlinear observer presented in Ref. [5] is used for attitude estimation; however, two slight modifications are introduced. The first one incorporates the angular velocity model in Eq. (23) and the second includes a model of a potential bias, represented by $\mathbf{b}^{m}$, in the quaternion differential equation to include any offset of the sensor, which may even be the computation of the focal distance. A first order model is chosen for this bias, given by

$$
\dot{\mathbf{b}}^{m}=-M \mathbf{b}^{m}+\boldsymbol{\eta}_{b}
$$

where the $M$ is a diagonal positive definite matrix which represents the time constant of the process, and $\boldsymbol{\eta}_{b}$ is a Gaussian noise.

The proposed observer follows the same concept of the original work of Luenberger, ${ }^{13}$ by introducing feedback terms to drive the error between the measured and estimated quantities to zero with some rate of convergence. The dynamics of the observer are given by

$$
\begin{gathered}
\dot{\hat{\boldsymbol{\varrho}}}_{s}^{m}=\frac{1}{2}\left[\hat{q}_{o} I_{3 \times 3}+\left[\hat{\boldsymbol{\varrho}}_{s}^{m} \times\right]\right] \\
\times\left[\hat{\boldsymbol{\omega}}_{m s}^{m}+\hat{\mathbf{b}}+K_{v} \delta \varrho \operatorname{sign}\left(\delta q_{o}\right)\right] \\
\dot{\hat{q}}_{o}=-\frac{1}{2}\left(\hat{\boldsymbol{\varrho}}_{s}^{m}\right)^{T}\left[\hat{\boldsymbol{\omega}}_{m s}^{m}+\hat{\mathbf{b}}+K_{v} \delta \varrho \operatorname{sign}\left(\delta q_{o}\right)\right] \\
\dot{\hat{\boldsymbol{\omega}}}_{m s}^{m}=-\Lambda \hat{\boldsymbol{\omega}}_{m s}^{m}+\Delta \hat{\mathbf{T}}^{m}+K_{p} \delta \boldsymbol{\varrho} \operatorname{sign}\left(\delta q_{o}\right) \\
\Delta \dot{\hat{\mathbf{T}}}^{m}=-H \Delta \hat{\mathbf{T}}^{m}+K_{T} \delta \varrho \operatorname{sign}\left(\delta q_{o}\right) \\
\dot{\hat{\mathbf{b}}}^{m}=-M \hat{\mathbf{b}}^{m}+K_{b} \delta \boldsymbol{\varrho} \operatorname{sign}\left(\delta q_{o}\right)
\end{gathered}
$$

where $K_{v}, K_{p}, K_{T}$ and $K_{b}$ are positive definite matrices. The sign function ensures that the smallest possible angle is chosen between the two equivalent rotations angles described by $\phi$ and $2 \pi-\phi$.

The quaternion error between the truth $(\mathbf{q})$ and estimate $(\hat{\mathbf{q}})$ is defined, following Ref. [4], as $\delta \tilde{\mathbf{q}}=\mathbf{q} \otimes \hat{\mathbf{q}}^{-1}$, and the error dynamics are given by

$$
\begin{gathered}
\delta \dot{\tilde{q}}=\dot{\mathbf{q}} \otimes \hat{\mathbf{q}}^{-1}+\mathbf{q} \otimes \dot{\hat{\mathbf{q}}}^{-1} \\
=\frac{1}{2}[\boldsymbol{\omega} \otimes \delta \mathbf{q}-\delta \tilde{\mathbf{q}} \otimes \hat{\boldsymbol{\omega}}] \\
\delta \dot{\tilde{q}}=\frac{1}{2} \delta \tilde{\mathbf{q}} \otimes(\boldsymbol{\omega}-\hat{\boldsymbol{\omega}})=\frac{1}{2} \delta \tilde{\mathbf{q}} \otimes \tilde{\boldsymbol{\omega}}
\end{gathered}
$$

where the superscript $(\tilde{\cdot})$ indicates the error between the truth and the estimate. Using Eq. (38) the error dynamics can be shown to be given by

$$
\begin{gathered}
\delta \dot{\tilde{\varrho}}=\frac{1}{2}\left[\delta \tilde{q}_{o} I_{3 \times 3}+[\delta \tilde{\boldsymbol{\varrho}} \times]\right] \\
\times\left[\tilde{\boldsymbol{\omega}}_{m s}+\tilde{\mathbf{b}}-K_{v} \delta \tilde{\varrho} \operatorname{sign}\left(\delta \tilde{q}_{o}\right)\right] \\
\delta \dot{\tilde{q}}_{o}=-\frac{1}{2}(\delta \tilde{\boldsymbol{\varrho}})^{T}\left[\tilde{\boldsymbol{\omega}}_{m s}+\tilde{\mathbf{b}}-K_{v} \delta \tilde{\varrho} \operatorname{sign}\left(\delta \tilde{q}_{o}\right)\right] \\
\dot{\tilde{\boldsymbol{\omega}}}_{m s}=-\Lambda \tilde{\boldsymbol{\omega}}_{m s}-\Delta \tilde{\mathbf{T}}-K_{p} \delta \tilde{\varrho} \operatorname{sign}\left(\delta \tilde{q}_{o}\right) \\
\Delta \dot{\tilde{\mathbf{T}}}=-H \Delta \tilde{\mathbf{T}}-K_{T} \delta \tilde{\varrho} \operatorname{sign}\left(\delta \tilde{q}_{o}\right) \\
\dot{\tilde{\mathbf{b}}}=-M \tilde{\mathbf{b}}-K_{b} \delta \tilde{\varrho} \operatorname{sign}\left(\delta \tilde{q}_{o}\right)
\end{gathered}
$$

In the above expressions, the indication of frames has been deleted to simplify the notation, but all vectors are expressed in the master frame, and the quaternion error describes the rotation between $\hat{m}$ and $m$. The system clearly has two equilibrium points for the set of the angular vectors, $\left[\tilde{\boldsymbol{\omega}}, \delta q_{o}, \delta \varrho, \Delta \mathbf{T}, \mathbf{b}\right]=$ $\left[\mathbf{0}_{1 \times 3}, \pm 1, \mathbf{0}_{1 \times 3}, \mathbf{0}_{1 \times 3}, \mathbf{0}_{1 \times 3}\right]$.

The extended Kalman filter can be used to also estimate the relative attitude and position from line of sight observations. However, a proof of global asymptotic stability using the extended Kalman filter even for the attitude only problem is difficult to derive. Global asymptotic stability for the entire problem involving attitude and position for the observer derived in this section has not been shown to date; however, an analysis of the stability of the observer design can be shown by dividing the entire problem into independent attitude and position cases. Although this approach does not prove global stability, it does provide some measure of local stability for each problem. A Lyapunov stability approach and contraction theory are used in each case.

The next step is to analyze the stability of the error equation. Because the system is nonlinear and nonautonomous, Lyapunov's approach is used. Based on the candidate Lyapunov function used in Ref. [5], the following function is chosen

$$
\begin{aligned}
& V=\frac{1}{2}\left(\tilde{\boldsymbol{\omega}}^{T} K_{p}^{-1} \tilde{\boldsymbol{\omega}}+\tilde{\mathbf{b}}^{T} K_{b}^{-1} \tilde{\mathbf{b}}\right) \\
& + \begin{cases}\left(\delta q_{o}-1\right)^{2}+\delta \varrho^{T} \delta \varrho & \text { if } \delta q_{o} \geq 0 \\
\left(\delta q_{o}+1\right)^{2}+\delta \varrho^{T} \delta \varrho & \text { if } \delta q_{o}<0\end{cases}
\end{aligned}
$$

From the definition of quaternion, the following two relations are true: $\delta q_{o}^{2}+\delta \varrho^{T} \delta \varrho=1$ and $\delta q_{o} \delta \dot{q}_{o}+\delta \boldsymbol{\varrho}^{T} \delta \dot{\varrho}=$ 0 . Taking the time derivative of the candidate Lyapunov function, the following expression is obtained

$$
\begin{aligned}
\dot{V} & =\tilde{\boldsymbol{\omega}}^{T} K_{p}^{-1} \dot{\tilde{\boldsymbol{\omega}}}+\tilde{\mathbf{b}}^{T} K_{b}^{-1} \dot{\tilde{\mathbf{b}}} \\
& +\left\{\begin{array}{l}
-2 \delta \dot{q}_{o} \quad \text { if } \delta q_{o} \geq 0 \\
2 \delta \dot{q}_{o} \quad \text { if } \delta q_{o}<0
\end{array}\right.
\end{aligned}
$$

Substituting Eqs. (40), (41) and (43) into Eq. (45) 
yields

$$
\dot{V}=-\tilde{\boldsymbol{\omega}}^{T} K_{p}^{-1} \Lambda \tilde{\boldsymbol{\omega}}-\tilde{\mathbf{b}}^{T} K_{b}^{-1} M \tilde{\mathbf{b}}-\delta \varrho^{T} K_{v} \delta \varrho
$$

The function $V$ is clearly always negative, so $V$ is indeed a Lyapunov function and the stability has been proved. Therefore, Eq. (46) assures that $\|\delta \varrho\| \rightarrow 0$, and since $\delta q_{o}^{2}+\delta \varrho^{T} \delta \varrho=1$ the magnitude of the scalar part converges exponentially with $\left|\delta q_{o}\right| \rightarrow 1$.

\section{Navigation Observer}

The observers for the relative position and relative linear velocity are given by

$$
\begin{gathered}
\dot{\hat{\mathbf{r}}}=\hat{\mathbf{v}}-K_{p} d \mathbf{z} \\
\dot{\hat{\mathbf{v}}}=f(\hat{\mathbf{r}}, \hat{\mathbf{v}})-K_{v} d \mathbf{z}
\end{gathered}
$$

where $f(\cdot)$ is the right hand side of Eq. (14), $\mathbf{r}$ is the relative position vector, and $\mathbf{v}$ is the relative linear velocity vector. The minus signs in Eqs. (47) and (48) are due to the definition of $d \mathbf{z}$. The constant gains $K_{p}$ and $K_{v}$ are positive definite matrices (usually diagonal).

The error equations are directly calculated as

$$
\begin{gathered}
\dot{\tilde{\mathbf{r}}}=\tilde{\mathbf{v}}+K_{p} d \mathbf{z} \\
\dot{\tilde{\mathbf{v}}}=f(\mathbf{r}, \mathbf{v})-f(\hat{\mathbf{r}}, \hat{\mathbf{v}})+K_{v} d \mathbf{z}
\end{gathered}
$$

To prove observer convergence the right hand side of Eq. (50) must be bounded. Both functions $f(\mathbf{r}, \mathbf{v})$ and $f(\hat{\mathbf{r}}, \hat{\mathbf{v}})$ are continuous so that a derivative always exists. The distance between both functions is given by the mismatch between the true and estimated quantities. Lipschitz's condition is used to quantify this mismatch with $[f(\mathbf{x})-f(\hat{\mathbf{x}})] \leq K\|\tilde{\mathbf{x}}\|$, where the vector $\mathbf{x}=[\mathbf{r}, \mathbf{v}]^{T}$ represents the state vector. The value of $K$ is calculated using the mean value inequality, i.e. $K=\sup \left\|f^{\prime}(\mathbf{x})\right\|$, where the supremum is calculated in the range of possible variations of the state vector in a limited time span. For the case of $K \leq 1$, the observer can be defined by a contraction mapping. ${ }^{14}$ To complete the contraction mapping, two operators are defined: $T(\mathbf{x})=\int_{t_{o}}^{t_{f}} f[\mathbf{x}(\tau)] d \tau$, and $T(\hat{\mathbf{x}})=\int_{t_{o}}^{t_{f}} f[\hat{\mathbf{x}}(\tau)] d \tau$. Using the Lipschitz condition, the sequential estimation process after $n$ time steps gives the following inequality

$$
\|T(\mathbf{x})-T(\hat{\mathbf{x}})\| \leq \frac{K^{n} \Delta t^{n}}{n !}\|\tilde{\mathbf{x}}\|
$$

where $\Delta t$ is the sampling interval. In the attitude observer the observation is modeled by a function of a rotation matrix or a quaternion error. In the position case the observation is modeled using the position vector $\mathbf{r}$. The relation between the measurement residual and the position can be written as $d \mathbf{z}=-\kappa \tilde{\mathbf{r}}$, where $\kappa$ is a positive definite matrix. This approach assumes that a linear relationship between the residual and position error exists, which is valid since the projection of
Table 1 Orbital Elements of the Master Satellite.

$\begin{array}{cc}\text { Semimajor axis } & a=6,878 \mathrm{~km} \\ \text { Eccentricity } & e=0 \\ \text { Inclination } & i=50^{\circ} \\ \text { Node right ascension } & \Omega=5^{\circ} \\ \text { Argument of perigee } & \omega=10^{\circ} \\ \text { Mean anomaly } & M=8^{\circ}\end{array}$

line of sight on the focal plane is an affine function of the relative position. We note that this relation is used only in the analysis; the observer given in Eq. (48) is used in the actual implementation. The position and velocity errors can now be re-written as

$$
\left[\begin{array}{c}
\dot{\tilde{\mathbf{r}}} \\
\dot{\mathbf{v}}
\end{array}\right]=\left[\begin{array}{ll}
-K_{p} \kappa+K & I_{3 \times 3} \\
-K_{v} \kappa+K & 0_{3 \times 3}
\end{array}\right]\left[\begin{array}{c}
\tilde{\mathbf{r}} \\
\tilde{\mathbf{v}}
\end{array}\right]
$$

where the gains are selected to have $K<K_{p} \kappa$ and $K<K_{v} \kappa$. The linear system is now exponentially stable, where the proof can be found in Ref. [5].

\section{$\underline{\text { Simulation }}$}

The orbital elements used in the simulation of the master satellite are shown in Table 1. A small initial condition perturbation of these elements is used to simulate the motion of the secondary satellite. A plot of the relative motion between both satellites for an 80 second simulation is shown in Figure 5. The true inertia matrices of both satellites is given by

$$
I_{s}=I_{m}=\operatorname{diag}[100,120,130] \mathrm{N}-\mathrm{m}-\mathrm{s}^{2}
$$

In the observer the following inertia matrices are used:

$$
I_{s}=I_{m}=\operatorname{diag}[110,115,140] \mathrm{N}-\mathrm{m}-\mathrm{s}^{2}
$$

The true relative initial angular velocity is given by

$$
\boldsymbol{\omega}=[0.065,0.048,0.03]^{T} \mathrm{deg} / \mathrm{sec}
$$

The relative angular velocity trajectory is computed by integrating the following equation

$$
\dot{\omega}=\lambda I_{s}^{-1} \omega
$$

where $I_{s}$ is the true inertia and $\lambda=0.02$. A noise of $f / 3000$ is assumed for each measurement on the focal plane and four beacons have been placed on the secondary satellite at a distance of 1 meter from the mass center, along each coordinate axis. The fourth beacon is placed at $[1,1,1]^{T}$ in the secondary frame.

The observer described in the last section is implemented for state estimation from the line-of-sight measurements. The initial condition angular error is a rotation of about $15^{\circ}$ along each of the coordinates axes. The initial angular velocity has 50 percent errors from Eq. (55). The initial position condition 10 percent from the true value and the initial linear velocity condition is 30 percent from the true value. The 


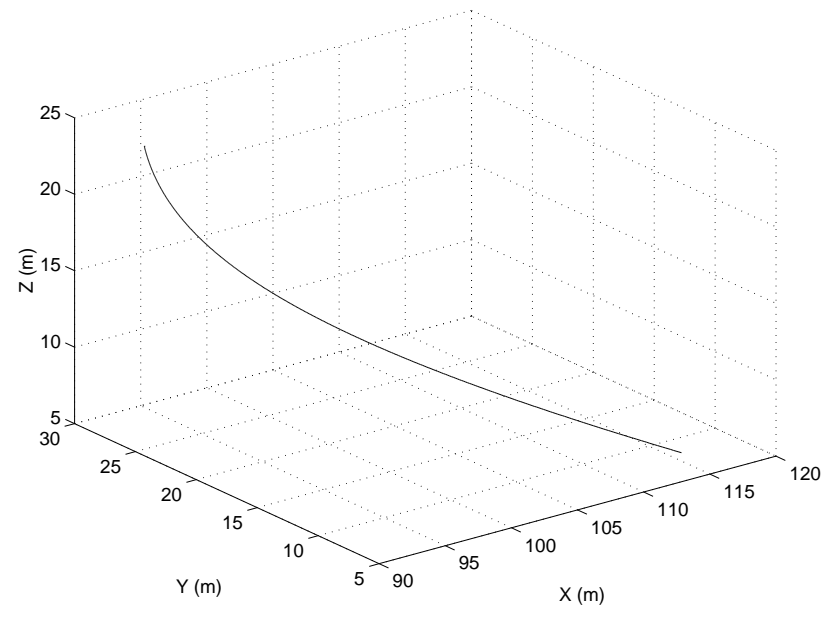

Fig. 5 True Relative Position Between Satellite


Fig. 6 Attitude Errors

sampling rate is $4 \mathrm{~Hz}$. Figures 6-9 show attitude and angular velocity errors, and position and linear velocity errors for the estimator. The relative distance along the $X$ axis is almost three times the distance along the other two axes (around 94 meters against 30 meters). This difference can be observed in the oscillation of the attitude error in roll, which is intuitively correct. The roll angle error is within 0.3 degrees, and the pitch and yaw angles are within 0.05 degrees. The position error in all three axis is within $1 \mathrm{~cm}$. Also, the velocities are well estimated using the observer.

\section{Conclusions}

An observer based system has been presented as an alternative to the extended Kalman Filter for formation flying navigation of spacecraft. The measurements were assumed to be given by line of sight observations using a novel sensing approach involving LED beacons and position sensing technology in the focal plane. The observer design utilizes a simple term in the attitude estimator to insure that convergence is achieved in the fastest time possible, and stability has
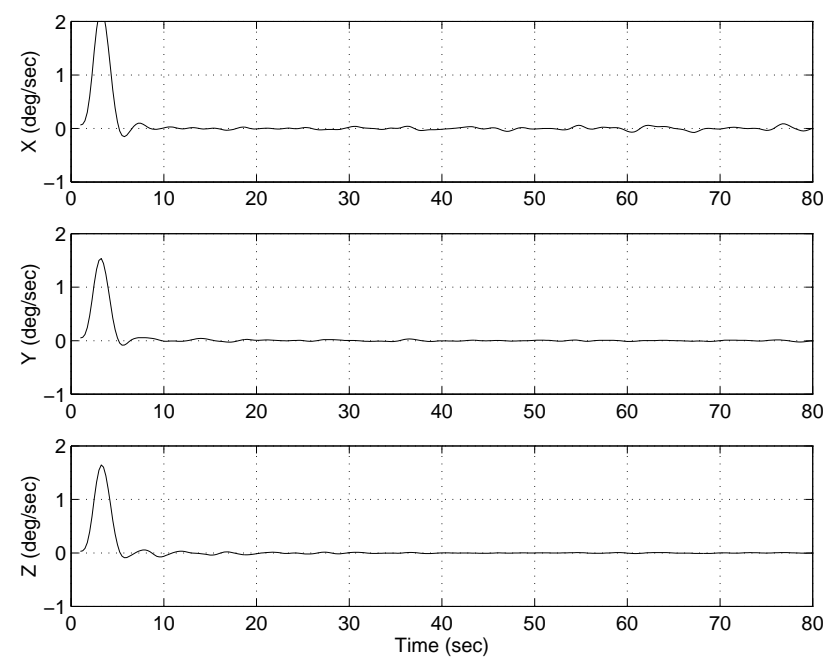

Fig. 7 Angular Velocity Errors
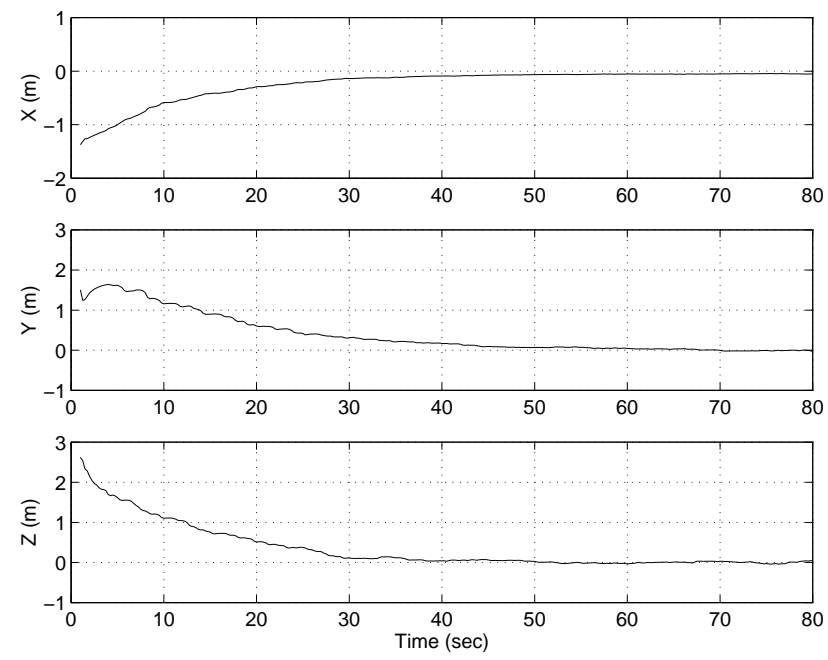

Fig. 8 Position Errors


Fig. 9 Linear Velocity Errors 
been proven using a local Lyapunov analysis. The relative position and linear velocity observer has been proven to meet quantitative convergence using a contraction mapping analysis. Simulation results have shown that accurate relative attitude and position estimation is possible.

\section{Acknowledgement}

This work was supported under a NASA grant (NCC 5-448), under the supervision of Dr. F. Landis Markley at NASA-Goddard Space Flight Center, and from a Texas Higher Education Coordinating Board Grant (000512-0004-1999) and Air Force Office of Sponsored Research grant (32525-57200). The authors wish to thank Dr. Markley for many helpful suggestions and comments.

\section{References}

${ }^{1}$ Corazzini, T., Robertson, A., Adams, J. C., Hassibi, A., and How, J. P., "GPS Sensing for Spacecraft Formation Flying," Proceedings of the 1997 ION-GPS, Kansas City, MO, Sept. 1997, pp. 735-744.

${ }^{2}$ Purcell, G., Kuang, D., Lichten, S., Wu, S.-C., and Young, L., "Autonomous Formation Flyer (AFF) Sensor Technology Development," 21st Annual AAS Guidance and Control Conference, Breckenridge, CO, Feb. 1998, AAS Paper 98-062.

${ }^{3}$ Junkins, J. L., Hughes, D. C., Wazni, K. P., and Pariyapong, V., "Vision-Based Navigation for Rendezvous, Docking and Proximity Operations," 22nd Annual AAS Guidance and Control Conference, Breckenridge, CO, February 1999, AAS Paper 99-021.

${ }^{4}$ Lefferts, E. J., Markley, F. L., and Shuster, M. D., "Kalman Filtering for Spacecraft Attitude Estimation," Journal of Guidance, Control, and Dynamics, Vol. 5, No. 5, Sept.-Oct. 1982, pp. 417-429.

${ }^{5}$ Salcudean, S., "A Globally Convergent Angular Velocity Observer for Rigid Body Motion," IEEE Transactions on Automatic Control, Vol. 36, No. 12, Dec. 1991, pp. 1493-1497.

${ }^{6}$ Wrigley, W., Hollister, W., and Denhard, W., Gyroscopic Theory, Design, and Instrumentation, MIT Press, Cambridge, MA, 1969.

${ }^{7}$ Light, D. L., "Satellite Photogrammetry," Manual of Photogrammetry, edited by C. C. Slama, chap. 17, American Society of Photogrammetry, Falls Church, VA, 4th ed., 1980.

${ }^{8}$ Crassidis, J. L., Alonso, R., and Junkins, J. L., "Optimal Attitude and Position Determination From Line of Sight Measurements," The Richard H. Battin Astrodynamics Conference, College Station, TX, March 2000, AAS Paper 00-268.

${ }^{9}$ Shuster, M. D., "A Survey of Attitude Representations," Journal of the Astronautical Sciences, Vol. 41, No. 4, Oct.-Dec. 1993, pp. 439-517.

${ }^{10}$ Battin, R. H., An Introduction to the Mathematics and Methods of Astrodynamics, American Institute of Aeronautics and Astronautics, Inc., New York, NY, 1987.

${ }^{11}$ Bate, R. R., Mueller, D. D., and White, J. E., Fundamentals of Astrodynamics, Dover Publications, New York, NY, 1971

${ }^{12}$ Tschauner, J., "Elliptic Orbit Rendezvous," AIAA Journal, Vol. 5, No. 6, June 1967, pp. 1110-1113.

${ }^{13}$ Luenberger, D. G., "Observers for Multivariable Systems," IEEE Transactions on Automatic Control, Vol. 11, No. 2, April 1966, pp. 190-197.

${ }^{14}$ Luenberger, D. G., Optimization by Vector Space Methods, John Wiley \& Sons, Inc., New York, NY, 1969. 